\title{
Estigma personal y actividades psicoeducativas en pacientes de psiquiatría del Complejo Hospitalario Universitario de Canarias
}

Personal Stigma and Psychoeducational Activities in Patients of Psychiatric Departments from Complejo Hospitalario Universitario de Canarias

\section{María Jesús Bello Hernández', Laura Rodríguez Frías', Ana Adey Rodríguez Fernández², Ruth Auxiliadora Díaz Melián', Miguel Ángel Rodríguez García ${ }^{3}$}

'Unidad de Internamiento Breve del Complejo Hospitalario Universitario de Canarias (CHUC). Tenerife, España.

${ }^{2}$ Unidad de Rehabilitación Activa del Complejo Hospitalario Universitario de Canarias (CHUC). Tenerife, España.

${ }^{3}$ Unidad de Salud Mental Comunitaria de La Laguna. Tenerife, España.

Contacto: susabhdez@gmail.com

Fecha de recepción: 6 de mayo de 2018 / Fecha de aceptación: 1 de septiembre de 2018

\begin{abstract}
Resumen
Introducción: El estigma hace referencia a las actitudes y creencias que conducen a las personas a rechazar, evitar y temer a aquellos a quienes perciben diferentes. Afecta a las personas con enfermedad mental, provocando implicaciones negativas en diferentes áreas de su vida, así como discriminación por parte de la sociedad y auto-rechazo (autoestigma). El presente trabajo tiene como objetivo describir la presencia del estigma personal en pacientes con Trastorno Mental Grave y poner de manifiesto la necesidad de implantar actividades psicoeducativas para ayudar a vencer el autoestigma y afrontar situaciones sociales estigmatizantes.

Metodología: Se trata de un estudio descriptivo transversal realizado en enero de 2018. Se han recabado 45 sujetos, procedentes de diferentes unidades de psiquiatría del Complejo Hospitalario Universitario de Canarias (Tenerife). El instrumento de medida utilizado fue la escala validada ISMI (Internalized Stigma of Mental IIIness), utilizando una versión es español.

Resultados: Un total de un $46 \%$ de pacientes dieron positivo en estigma personal y un 52,67\% presentaron resiliencia. El autoestigma se manifiesta en un $45 \%$ de los participantes. A pesar de que más de la mitad de participantes muestra resiliencia, aún se sienten discriminados por parte de la sociedad, lo que posiblemente influya en que más de la mitad de pacientes de positivo en aislamiento social. Se ha elaborado un taller psicoeducativo, donde los participantes han manifestado la necesidad de más actividades y atención psicológica relacionada con el tema en cuestión, así como proseguir en el intento de educar a la población general.

Conclusiones: Estimamos oportuno la implantación de actividades y talleres psicoeducativos en los servicios de salud mental, que permitan ayudar al paciente a desarrollar estrategias para la desaparición de los sentimientos relacionados con el autoestigma y para hacer frente a situaciones socialmente estigmatizantes.
\end{abstract}

Palabras clave: estigma personal, autoestigma, Trastorno Mental Grave, psicoeducación.

\begin{abstract}
Introduction: The stigma refers to attitudes and beliefs which move people to reject, avoid and fear someone who are perceived different. It affects people with mental illness, causing negatives implications in their lives, discrimination and rejection from people and society that surround them and the self-rejection (self-stigma). The aim of this research is to describe the presence of personal stigma on patients with serious mental disorders and highlight the need to introduce psychoeducational activities to inform and help to defeat self-stigma and confront social situations that generate stigma.

Methodology: It is a descriptive transversal research done in January 2018. 45 patients have been recruited from different psychiatric departments of the Complejo Hospitalario Universitario de Canarias (Tenerife). The measuring instrument used was the validated scale ISMI (Internalized Stigma of Mental Illness), using a Spanish version.

Results: As a result $46 \%$ of patients gave a positive result on self- stigma, and $52.67 \%$ showed resilience. Despite this
\end{abstract}


fact, they still feel discriminated and rejected by society. Self-stigma is manifested in $45 \%$ of participants. A psychoeducational seminar has been held, obtaining opinions where participants have expressed the need for more activities and psychological attention, as well as the education of society.

Conclusions: We may wish to highlight the need to carry out psychoeducational activities and seminars in mental health services, which inform and help to defeat self-stigma and confront social situations that generate stigma.

Keywords: personal stigma, self-stigma, serious mental disorders, psychoeducation.

\section{Introducción}

El estigma hace referencia a las actitudes y creencias que conducen a las personas a rechazar, evitar y temer a aquellos a quienes perciben diferentes ${ }^{1}$. La realidad del estigma social afecta a las personas con enfermedad mental, provocando implicaciones negativas en su calidad de vida, tanto debidas a la discriminación y rechazo de quienes le rodean y de la sociedad, como debido a su autorechazo (autoestigma o estigma internalizado $)^{2}$. En este sentido, las personas que sufren de alguna enfermedad mental tienden a tener prejuicios contra ellos mismos y a estar de acuerdo con los estereotipos típicos que se asocian a la psicopatología. Livingston y Boyd hallan que las variables psicosociales tales como esperanza, autoestima, empoderamiento, autoeficacia, calidad de vida, y variables sociales como apoyo social e integración social se relacionan significativamente y generalmente de manera inversa con el autoestigma. En relación con las variables psiquiátricas concluyen que hay una asociación negativa con la adherencia al tratamiento y positiva entre la severidad de los síntomas y el estigma internalizado ${ }^{3}$.

Las personas que padecen una enfermedad mental consideran que existe un gran desconocimiento sobre los trastornos mentales y un fuerte estigma, sintiéndose discriminados en distintas áreas de funcionamiento. Esto puede llevar al ocultamiento de su enfermedad y al aislamiento ${ }^{4}$. Más de la mitad de las personas con trastorno mental manifiestan haber sido tratadas injustamente en algún momento de su vida debido a tal condición ${ }^{5}$. La visión subjetiva del que padece la enfermedad en cuanto a cómo es percibido por los otros, especialmente los más próximos, y sobre su enfermedad, influirá directamente en su integración en la comunidad a todos los niveles: vivienda, relaciones sociales, trabajo, etc. ${ }^{3}$.

En Europa destaca el estudio GAMIAN-Europa (Global Alliance of Mental Illness Advocacy Networks) que estudia los niveles de autoestigma, de resistencia al estigma y de discriminación percibida, los cuales conforman el estigma personal. Orientan a que las variables más relacionadas con el estigma internalizado son la autoestima/autoeficacia, empoderamiento, conocer y estar de acuerdo con el diagnóstico y tener un alto número de contactos sociales ${ }^{6}$.

El estigma social no se identifica de igual forma en todas las enfermedades mentales, siendo en los Trastornos Mentales Graves donde más se manifiesta, con comportamientos de miedo y rechazo por parte de la sociedad. Existe aún un gran desconocimiento sobre los distintos tipos de enfermedades mentales y de la diversidad de las personas afectadas por las mismas ${ }^{2}$.

Es preciso distinguir entre el estigma percibido como tal y el temor a ser discriminado. La aprehensión conduce a ocultar o disfrazar los síntomas, lo que implica el diferimiento de un diagnóstico oportuno y la postergación indefinida del tratamiento, a veces con consecuencias muy serias para el curso y pronóstico de la enfermedad mental severa y persistente. Con el miedo al rechazo y a la discriminación, los pacientes que han sido hospitalizados por problemas mentales con frecuencia actúan en forma defensiva y evitan el contacto con los servicios de salud ${ }^{7}$. Siguiendo esta línea, diversos estudios definen que el autoestigma queda afectado fundamentalmente por la calidad de las relaciones sociales y por la sensación de tener dificultades cognitivas ${ }^{6,8,9}$.

En cuanto al estigma social, son varios los estudios que concluyen que aún se mantienen los estereotipos negativos relacionados con las enfermedades mentales, como la peligrosidad y la imprevisibilidad, así como las actitudes de rechazo o discriminación ${ }^{10,11}$. Así mismo, continúan ciertas actitudes de paternalismo y sobreprotección hacia las personas con enfermedad mental $^{12}$.

Por todo esto, estimamos necesario hacer estudios para determinar el estigma personal y así realizar programas de intervención adecuados para dotar al sujeto de recursos y estrategias de afrontamiento ante tales 
situaciones estigmatizadoras ${ }^{3}$. Esto no sería posible sin instrumentos adecuados para la valoración del estigma personal. La escala Internalized Stigma of Mental Illness (ISMI) es uno de los instrumentos más utilizados para valorar el estigma de la enfermedad mental, la cual está compuesta por 29 ítems y 5 subescalas ${ }^{13}$. Para el presente estudio hemos seleccionado una versión en español, en la cual se midieron las propiedades psicométricas dando buenos resultados, con valores adecuados de fiabilidad test-retest y de consistencia interna mediante el alfa de Cronbach ${ }^{14}$.

Existen diferentes formas de contribuir a la lucha contra el estigma en salud mental. Un ejemplo es el Proyecto Saltando Muros, desarrollado por Esther Sanz y César M. Estévez (Psicóloga Clínica y Enfermero especialista en Salud Mental de Tenerife), cuyo eje central son las personas afectadas por Trastorno Mental Grave. A través de su blog, que se encuentra inactivo en este momento, se engloban diferentes conceptos como la recuperación, el respeto de los derechos humanos, el empoderamiento y la reclamación de un espacio en salud mental para la voz de las personas afectadas, donde poder demostrar que tienen el derecho de tomar las riendas de su vida, desarrollar sus potencialidades y contribuir con sus aportaciones a la sociedad $^{15}$.

El objetivo del presente trabajo es describir la presencia del estigma personal en pacientes con Trastorno Mental Grave de diferentes servicios de psiquiatría pertenecientes a la gestión del Complejo Hospitalario Universitario de Canarias (Tenerife), así como relacionarlo con variables sociodemográficas y clínicas. Por otro lado, poner de manifiesto la necesidad de implantar actividades psicoeducativas para informar y ayudar a vencer el autoestigma y a afrontar situaciones sociales estigmatizantes.

\section{Metodología}

Se trata de un estudio descriptivo transversal, realizado durante el mes de enero de 2018. A través de un muestreo consecutivo se han recabado los sujetos del estudio. Los criterios de inclusión fueron presentar un Trastorno Mental Grave y tener una edad de entre 1865 años, no incluyendo a aquellas personas con dificultades cognitivas evidentes que impidiesen comprender las cuestiones a realizar en el momento de la valoración. Los participantes dieron su consentimiento verbal para la realización de las encuestas tras recibir la información pertinente, las cuales se hicieron de forma anónima y se identificaron correlativamente mediante una numeración.

La muestra estuvo compuesta por un total de 45 sujetos (18 mujeres y 27 hombres), con un rango de edad medio entre 30-49 años. En cuanto al diagnóstico, hemos obtenido un total de 71,12\% de trastornos psicóticos (75\% diagnosticados de Esquizofrenia), $24,45 \%$ trastornos afectivos $(63,64 \%$ diagnosticados de Trastorno Bipolar) y un 4,45\% de trastornos de la personalidad. Todos los pacientes pertenecían a unidades de psiquiatría correspondientes a la gestión del Hospital Universitario de Canarias, siendo un 22,22 \% ambulatorios (Unidad de Salud Mental de La Laguna) y un $77,78 \%$ hospitalizados $(28,57 \%$ de la Unidad de Rehabilitación Activa y un $71,43 \%$ de la Unidad de Internamiento Breve). El nivel educativo predominante fueron los estudios primarios, así como un nivel socioeconómico medio. En cuanto al inicio de la patología, más del 66,67\% de pacientes debutó con menos de 25 años y un 51,11\% contaba con más de 10 años de evolución de su patología.

El principal instrumento de medida fue la Escala validada ISMI (Internalized Stigma of Mental Illness), utilizando una versión en español propuesta en un estudio realizado en España14. Dicha escala está compuesta por 29 ítems que se agrupan en 5 subescalas: alienación, interiorización de estereotipos, aislamiento social, experiencias de discriminación y resistencia al estigma. Todos los ítems se valoran en una escala Likert del 1 al 4, siendo 1 (totalmente en desacuerdo), 2 (algo en desacuerdo), 3 (algo de acuerdo), 4 (totalmente de acuerdo).

Se utilizaron diferentes tipos de variables; por un lado, sociodemográficas (edad, sexo, nivel educativo, estatus socioeconómico, situación laboral), así como determinadas variables clínicas (edad de inicio de la patología, años de evolución, tipo de patología y número de ingresos hospitalarios). Por otra parte, se analizó las variables de autoestigma, estigma percibido y estigma experimentado a través de los resultados de la escala administrada.

En primer lugar, se estableció el cronograma a seguir para la elaboración del presente trabajo. Se aplicaron los criterios de inclusión/exclusión a los sujetos ingresados en Unidades de Hospitalización de Psiquiatría del Hospital Universitario de Canarias (Unidad de Internamiento Breve y Unidades de Rehabilitación Activa del Área Externa de Salud Mental de 


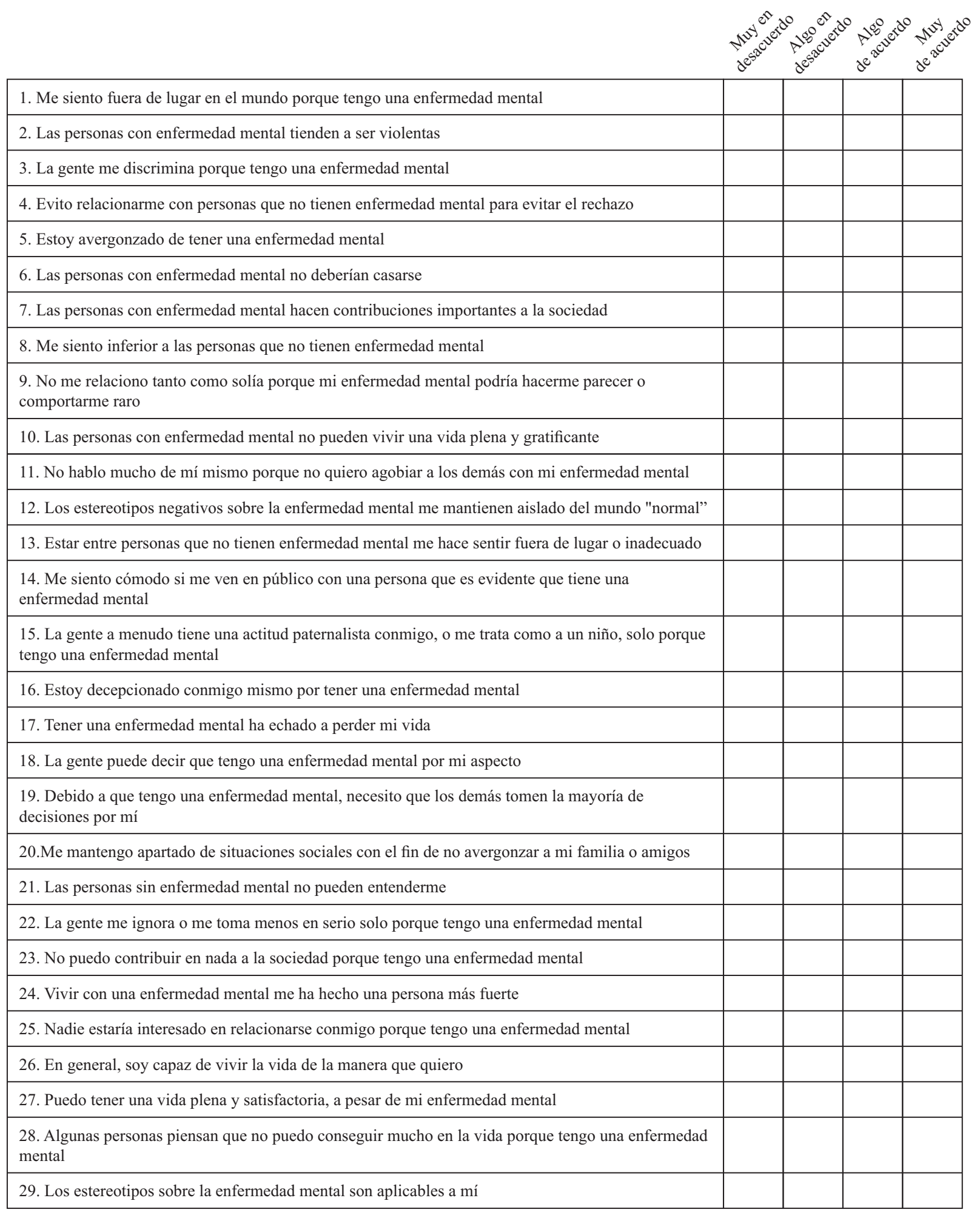

Tabla 1: Internalized Stigma of Mental Illness Scale (ISMI) - Versión en español 
Tenerife) y a aquellos que acudieron a la consulta de enfermería de la Unidad de Salud Mental de La Laguna entre las fechas anteriormente descritas, tras expresar su consentimiento. Las encuestas se pasaron in situ de forma anónima, siendo realizadas por 2 enfermeras especialistas en Salud Mental de la Unidad de Internamiento Breve, una enfermera de la Unidad de Rehabilitación Activa y un enfermero residente de Salud Mental en rotación por la Unidad de Salud Mental de la Laguna. Tras la realización de las encuestas, se han unificado los resultados obtenidos.

\section{Resultados}

Destacamos que un total de un $46 \%$ de pacientes dieron positivo en estigma personal. Por otro lado, un $52,67 \%$ de pacientes presentaron resiliencia. No se encontraron datos significativos respecto a la relación con las variables sociodemográficas y clínicas; aunque se comprobó que las mujeres, en general, estaban más estigmatizadas que los hombres.

En cuanto a las subescalas que engloba la encuesta utilizada, hemos obtenido que un 55,56\% de participantes dieron positivo en alienación, un $28,89 \%$ en interiorización de estereotipos, un $51,11 \%$ en aislamiento social y un $62,22 \%$ en experiencias de discriminación.

De las preguntas realizadas destacamos algunas como, por ejemplo, el sentirse fuera de lugar en el mundo por tener una enfermedad mental, donde el $66,77 \%$ estuvo de acuerdo. El $62,23 \%$ reconoció que la gente los discrimina por tener una enfermedad mental y el $55,56 \%$ se siente avergonzado por tenerla. Algo más del $66 \%$ de participantes prefieren no hablar de su psicopatología por no agobiar a los demás y reconocen que los estereotipos negativos sobre la enfermedad mental les hacen sentir fuera de lugar o inadecuados. Por otra parte, el 68,89\% admite que los demás suelen tener una actitud paternalista por el simple hecho de tener un trastorno mental. Un $40 \%$ de sujetos está al menos algo de acuerdo en que tener una enfermedad mental les ha arruinado la vida. En otra instancia, un $55,56 \%$ se siente incomprendido, ignorando o en desconsideración por parte de la sociedad. Hay que destacar que se evidencian sentimientos de incapacidad en al menos un 44,45 \%, al afirmar que han estado algo o muy de acuerdo en que necesitan que los demás tomen la mayoría de decisiones por ellos. La percepción del estigma social aflora en otros datos, por ejemplo, que el $62,23 \%$ reconocen que algunas personas piensan que no pueden conseguir mucho en la vida por tener una enfermedad mental.

En contraposición, hemos obtenidos algunos datos algo más positivos, generalmente relacionados con preguntas de "interiorización de estereotipos" o "capacidad de resistencia al estigma". Por ejemplo, un $82,2 \%$ opina que las personas con enfermedad mental no tienen por qué ser violentas. Un 73,33\% muestra su disconformidad con la afirmación de que "las personas con enfermedad mental no deberían casarse". Por otro lado, un 57,78\% generalmente no experimentan sentimientos de inferioridad y un 55,56\% rechaza estar decepcionado consigo mismo por tener una enfermedad mental. El 66,67\% opina que las personas con enfermedad mental pueden hacer importantes aportaciones a la sociedad.

Por último, se ha elaborado un taller psicoeducativo relacionado con el tema a abordar como propuesta para futuros estudios. Se han desarrollado varias sesiones del taller propuesto. Durante los mismos, se han originado diferentes líneas de debate entre los participantes. Hemos recabado datos subjetivos del mismo, para incluir a modo informativo en las conclusiones del presente trabajo.

\section{Discusión}

Al igual que en otras investigaciones anteriores, que sitúan la prevalencia del estigma personal entre el $36 \%$ y $67 \%, 6,16,17$, en el presente estudio se ha obtenido un $46 \%$. En cuanto a las puntuaciones obtenidas en cada subescala, podemos determinar que se ofrecen las puntuaciones más altas en cuanto al estigma experimentado y las más bajas en los estereotipos asumidos. Es decir, la mayoría de los pacientes no se identifican con los estereotipos sociales que se tienen de la enfermedad mental (el aspecto, la peligrosidad, las limitaciones para llevar a cabo una vida normal: casarse, contribuir a la sociedad, etc.). Sin embargo, a pesar de que algo más de la mitad de participantes muestra una alta capacidad de resistencia al estigma, la mayoría se sienten discriminados por parte de la sociedad, lo que posiblemente influya en que más de la mitad de pacientes dé positivo en aislamiento social.

Otro dato que destacar es la diferencia entre los distintos tipos de servicios, donde los pacientes hospitalizados mostraron un $44 \%$ de estigma personal, frente a un $50 \%$ de ambulatorios. En cuanto a la resistencia al estigma, mostraron menor capacidad los 
pacientes de la Unidad de Salud Mental. Este hecho podría deberse a que los pacientes ingresados suelen recibir mayor información y actividades relacionadas con la lucha contra el estigma, además de que la muestra obtenida en las unidades de hospitalización ha sido generalmente de pacientes más jóvenes y con menores repercusiones respecto a la evolución de la enfermedad.

El autoestigma se manifiesta a través de las subescalas de alienación y de la interiorización de estereotipos, dando como resultado que un $45 \%$ de participantes lo presenta. Este porcentaje nos parece preocupante, puesto que podría dar lugar a dificultades para la aceptación de la enfermedad y el seguimiento terapéutico, así como empeorar las expectativas de recuperación.

No obstante, no podemos afirmar que los resultados sean totalmente representativos, puesto que contamos con la limitación del tamaño y la variabilidad de la muestra utilizada, habiendo obtenido los sujetos del estudio a partir de un muestreo no probabilístico. A pesar de esto, se puede ofrecer una aproximación al problema, ya que los resultados obtenidos para la presencia de estigma personal se asemejan a otros estudios, así como los resultados en las subescalas.

\section{Conclusiones}

En definitiva, el estigma social continúa siendo un problema para las personas que padecen trastornos mentales. Este hecho puede desencadenar la aparición del autoestigma, en el que la misma persona que padece un trastorno mental asume los estereotipos y prejuicios sociales como propios, lo que contribuye a desarrollar una baja autoestima y a dificultar la toma de conciencia de enfermedad y la adherencia terapéutica. Por tanto, estimamos oportuno la implantación de actividades y talleres educativos en los servicios de salud mental, que permitan ayudar al paciente a desarrollar estrategias para la desaparición de los sentimientos relacionados con el autoestigma y para hacer frente a situaciones socialmente estigmatizantes.

Según la información obtenida tras la realización de los talleres psicoeducativos comentados, destacamos que:

- Existe un importante desconocimiento acerca del autoestigma en salud mental y sus repercusiones. Por ello, los participantes reclaman más atención psicológica y actividades psicoeducativas para mejorar el afrontamiento del mismo.

- La sobreprotección o infravaloración por parte de los familiares o allegados puede repercutir en la aparición del autoestigma, por lo que también se necesita actuar sobre el núcleo familiar.

- La mayoría de participantes están de acuerdo en que la sociedad y la educación son imprescindibles para conseguir un cambio en la estigmatización y demandan más actividades e información sobre patologías mentales.

- A pesar de que ha ido disminuyendo el estigma social, aún queda una importante labor para conseguir que desaparezca.

Para concluir, queremos resumir la esencia del presente trabajo en el siguiente discurso de un paciente, extraído de un artículo del Proyecto Saltando Muros: "Para mí ser enfermo mental no significa que seamos desaliñados, ya que hay mucha gente diagnosticada que es muy presumida; quiero que sepas que como en la vida misma, hay personas abandonadas y otras que son extremadamente limpias, no tenemos una apariencia determinada y no todos estamos aquí por drogas, o por pegar a alguien. Somos personas como otras y nos queda por hacer muchas cosas, tenemos capacidades como tienen todos, algunos somos brillantes, otros nos deprimimos ante una situación difícil; yo creo que ser enfermo mental no nos debe excluir de la sociedad, ni meternos en un saco, no somos ni siquiera un grupo, somos individuos con las mismas ganas de vivir y de ser felices y de no sufrir más que el resto de los mortales. Por eso yo os digo que os acerquéis sin miedo a nosotros, porque se puede descubrir una gran persona tanto en ti como en mí."18

\section{Bibliografía}

1. Disability Rights California [internet].California: Mental Health Service act California; 2012 [citado 17 marzo de 2018]. Disponible en: http://www.disabilityrightsca.org/pubs/CM0402.pdf

2. Fedeafes. La realidad del estigma social entre las personas con enfermedad mental. 1st ed. País Vasco; 2013.

3. Ochoa S, Martínez F, Ribas M, García-Franco M., López E, Villellas R, et. al. Estudio cualitativo sobre la autopercepción del estigma social en personas con esquizofrenia. Rev. Asoc. Esp. Neuropsiq., 2011;31 (111), 477-489.

4. Muñoz M, Pérez E, Crespo M. Estigma y enfermedad mental. Análisis del rechazo social que sufren las personas con enfermedad mental. 1st ed. Madrid: Complutense SA; 2009.

5. Obertament, Universitat Autónoma de Barcelona, Spora Sinergies. El estigma y la discriminación en Salud Mental. 1st ed. Cataluña: Apunts; 2016.

6. Brohan E, Elgie R, Sartorius N, Thornicroft G. Self-stigma, empowerment and perceived discrimination among people with schizophrenia in 14 European countries: The GAMIAN-Europe study. Schizophr Res, 2010; 122(13): 232-8. 
7. Duncan D. Mental Health University Institute - Research Centre McGill University Montreal, Canadá. Estigma y exclusión social en las enfermedad mentales. 2005; 6 (1), 3-14.

8. Muñoz M, Pérez-Sants E, Crespo M, Guillen A. Estigma y cognición social; estrategias para promover el cambio personal y social. Revista de psiquiatría y salud mental Hermilio Valdizán, Jul-Dic 2004; 5 (2): 3-16.

9. Garay M, Pousa V, Pérez L. La relación entre la percepción subjetiva del funcionamiento cognitivo y el autoestigma con la experiencia de recuperación de las personas con enfermedad mental grave. Rev.Asoc.Esp.Neuropsiq. 2014;34 (123): 459-475.

10. Wood L, Birtel M, Alsawy S, Pyle M, Morrison A. Public perceptions of stigma towards people with schizophrenia, depression, and anxiety. Psychiatry Res. 2014; 220: 604-8.

11. Aragonés E, López-Muntaner J, Ceruelo S, Basora J. Reinforcing stigmatization: Coverage of mental illness in Spanish newspapers. J Health Commun. 2014; 19: 1248-58.

12. Crespo M, Pérez Santos $E$, Muñoz M, Guillén A. Descriptive study of stigma associated with severe and persistent mental illness among the general population of Madrid (Spain). Community Ment Health J. 2008; 44: 393-403.

13. Ritsher JB, Otilingam PG, Grajales M. Internalized Stigma of Mental Illness: Psycometric properties of a new measure. Psychiatry Res. 2003; 121: 31-49.

14. Bengochea-Seco R, et al. Adaptación al español de la escala Internalized Stigma of Mental IIIness para valorar el estigma personal. Rev Psiquiatr Salud Ment (Barc). 2016. http://dx.doi.org/10.1016/j.rpsm.2016.01.007

15. Blog Saltando Muros [internet].Tenerife. 2009 [citado 26 marzo de $\left.\begin{array}{llll}2 & 0 & 1 & 8\end{array}\right] . \quad$ D is pon i b e e n : http://blogsaludmentaltenerife.blogspot.com.es/

16. Gerlinger $G$, Hauser $M$, de Hert $M$, Lacluyse $K$, Wampers $M$, Correll CU. Personal Stigma In schizophrenia Sprectrum Disorders: A systematic Review of Prevalence Rates, Correlates, impac and interventions. World Psychiatry. 2013., 12: 155-64.

17. West ML, Yanos PT, Smith SM, Roe D, Lysaker PH. Prevalence of internalized stigma among persons with severe mental illness. Stigma Res action. 2011;1:3-10

18. Sanz E. Internet como herramienta para compartir y construir miradas en torno a la psicosis: Saltando Muros y Psiquifotos. Intuiciones, delirios y terapias. XIX Curso Anual de Esquizofrenia. $2014 ; 3$ 